\title{
Description of an HIV-1 BC Recombinant Virus Identified in a Pediatric Patient in the City of São Paulo
}

\author{
Ana Carolina Mamana Fernandes de Souza ${ }^{1}$, Cristina Mendes de Oliveira ${ }^{1}$, Heloisa H.S. Marques ${ }^{2}$ and José Eduardo Levi ${ }^{1}$ \\ ${ }^{l}$ Virology Laboratory, Tropical Medicine Institute; ${ }^{2}$ Children Institute of Clinical Hospital of Medical School of São Paulo University (ICR-HC- \\ FMUSP); São Paulo, SP, Brazil
}

\begin{abstract}
This case report refers to a 10 -year-old HIV-1 infected patient, who was found to harbor a $\mathrm{BC}$ recombinant virus. This child lives in São Paulo and was infected by the mother-to-child route. Phylogenetic analyses revealed that this mosaic virus shares common breakpoints in the polymerase region with the recently published CRF31_BC. Key-Words: HIV-1, AIDS, children, circulating recombinant forms.
\end{abstract}

In 2007, 2.5 million children (0-15 years old) were living with HIV [1]. The Brazilian Ministry of Health reported that 589 new pediatric cases of AIDS were notified by June 2007 [2]. HIV-1 shows a high genetic diversity and is classified into three groups: $\mathrm{M}, \mathrm{O}$ and $\mathrm{N}$. The vast majority of strains found worldwide are classified into the $\mathrm{M}$ group, which includes nine subtypes (A, B, C, D, F, G, H, J and K) and at least 43 circulating recombinant forms (CRFs) [3,4]. In Brazil, at the beginning of the HIV epidemic only HIV-1 subtype B was observed. In 1994, a study identified co-circulation of a low proportion of HIV-1 F [5]. In the same study, the first recombinant virus circulating in Brazil, an HIV-1 BF, was observed [6]. Today, HIV-1 subtype B predominates, with co-circulation of HIV-1 F and $C[7,8]$. Recombinant viruses are responsible for approximately $15 \%$ of all HIV-1 infections [8]. However, a high frequency of HIV-1 subtype $\mathrm{C}$ has been documented in the southern part of the country [8-10]. A recent study showed that the Brazilian HIV-1 subtype C epidemic was the result of the introduction of a single founder strain of HIV-1 subtype $\mathrm{C}$ from Burundi, probably in the early 1980s [11].

Patient cells infected with HIV-1 that bear an RNA strand of one HIV-1 subtype and an RNA strand of another subtype are prone to make a recombinant proviral DNA during the reverse transcription process. The equally high frequency of subtype $\mathrm{B}$ and $\mathrm{C}$ viruses associated with that replication mechanism favors inter-subtype recombination events and different $\mathrm{BC}$ mosaics, including CRF31_BC, have been described $[10,12,13]$. CRF31_BC infections account for approximately $25 \%$ of the total HIV-1 viruses circulating in newly-infected individuals in Porto Alegre, the capital of the southernmost state $[10,12]$. Given the frequency of CRF31_BC in southern Brazil, we decided to investigate the introduction of this recombinant virus in São Paulo.

The case reported here is a boy, born in February 1997. He was infected by the mother-to-child transmission route and abandoned in an HIV children's care house, where he was Received on 15 October 2008; revised 29 January 2009.

Address for correspondence: Dr. José Eduardo Levi. Laboratório de Virologia, LIM 52. Instituto de Medicina Tropical da Universidade de São Paulo. Phone: 113062 2645. Fax 113063 2659. Rua Dr Enéas de Carvalho Aguiar 470, 2o andar. Zip code: 05403-000 São Paulo, SP Brazil. E-mail: dudilevi@usp.br.

The Brazilian Journal of Infectious Diseases 2009;13(1):67-69. (C) 2009 by The Brazilian Journal of Infectious Diseases and Contexto Publishing. All rights reserved. adopted. He was assisted first at another institution (Instituto de Infectologia Emílio Ribas, São Paulo, SP), where there is a record of HIV genotypic resistance testing in 2001. At that time, the test revealed resistance to AZT,3TC,DDI,D4T, moderate resistance to AZT $+3 \mathrm{TC}$ and Abacavir and sensitivity to Nevirapine and Efavirenz.

In 2003 the child was transferred to the outpatient clinic of the Instituto da Criança, Hospital das Clínicas, Faculdade de Medicina of São Paulo University (HC-FMUSP) Brazil, where he was maintained under a regimen of AZT $+3 \mathrm{TC} / \mathrm{Amprenavir/}$ Efavirenz up to May, 2007, at which time the CD4 count was 155 cells/ $\mu \mathrm{L}$ and the viral load 5,891 copies/mL. In July 2007, a blood sample was sent to the Virology Laboratory of the Instituto de Medicina Tropical for genotyping (antiretroviral genotypic resistance) under the HIV-1 Genotyping National Network (RENAGENO, PN DST/Aids, Brazilian Ministry of Health). Five milliliters of blood were collected in an EDTA tube, which was centrifuged at $2,000 \mathrm{x}$ g for 15 minutes. Plasma was collected and stored at $-70^{\circ} \mathrm{C}$, until use. HIV-1 protease (PR) and partial reverse transcriptase (RT) regions were sequenced with the ViroSeq ${ }^{\mathrm{TM}}$ Genotyping System kit (Celera Diagnostics, California, USA), according to manufacturer's instructions. Purified products were sequenced in an automated ABI Prism ABI 3100 Genetic Analyzer (Applied Biosystems, California, USA). All sequencing chromatograms obtained were assembled automatically (http:// www.ial.sp.gov.br/ci-bin/HIV/submissao) for subtype analysis. Strong evidence of resistance was found against all transcriptase inhibitors (NNRTI and NRTIs) except for TDF + 3TC and also against PIs, except for LPV/r and APV/r.

HIV-1 subtype was investigated with the NCBI website and REGA subtyping tool [14] and confirmed by phylogenetic analyses, as shown below. The sequence generated by the automated edition, a set of reference strains representative of all HIV-1 clades and the CRF31_BC reference strain, available in the Los Alamos HIV-1 database (www.hiv.lanl.gov), were aligned using Clustal W. In the phylogenetic analysis, we included $p o l$ fragments from two HIV-1 clade B and three HIV1 clade $\mathrm{C}$ isolates, previously analyzed in our laboratory. Alignment was manually corrected using BioEdit software (Ibis Therapeutics, USA). Phylogenetic analyses were performed with PAUP4b10 [15]. Neighbor Joining and Maximum Likelihood trees were constructed based on nucleotide substitution models determined by Modeltest v3.7 [16]. One thousand bootstrap replicates were used to assess 
Figure 1. Phylogenetic analysis of the HIV-1 pol region in São Paulo, Brazil. The tree was obtained using neighbor joining and a TVM $+\mathrm{I}+\mathrm{G}$ model. Reference HIV-1 clades were obtained from the Los Alamos Sequence database. Bootstrap values above $70 \%$ in key branches are depicted. The two HIV-1 clade $\mathrm{B}$ and the three HIV-1 clade $\mathrm{C}$ from that laboratory are in bold, named by their RENAGENO entry number. The HIV-1 study sequence is in bold and in a light-grey shaded box.

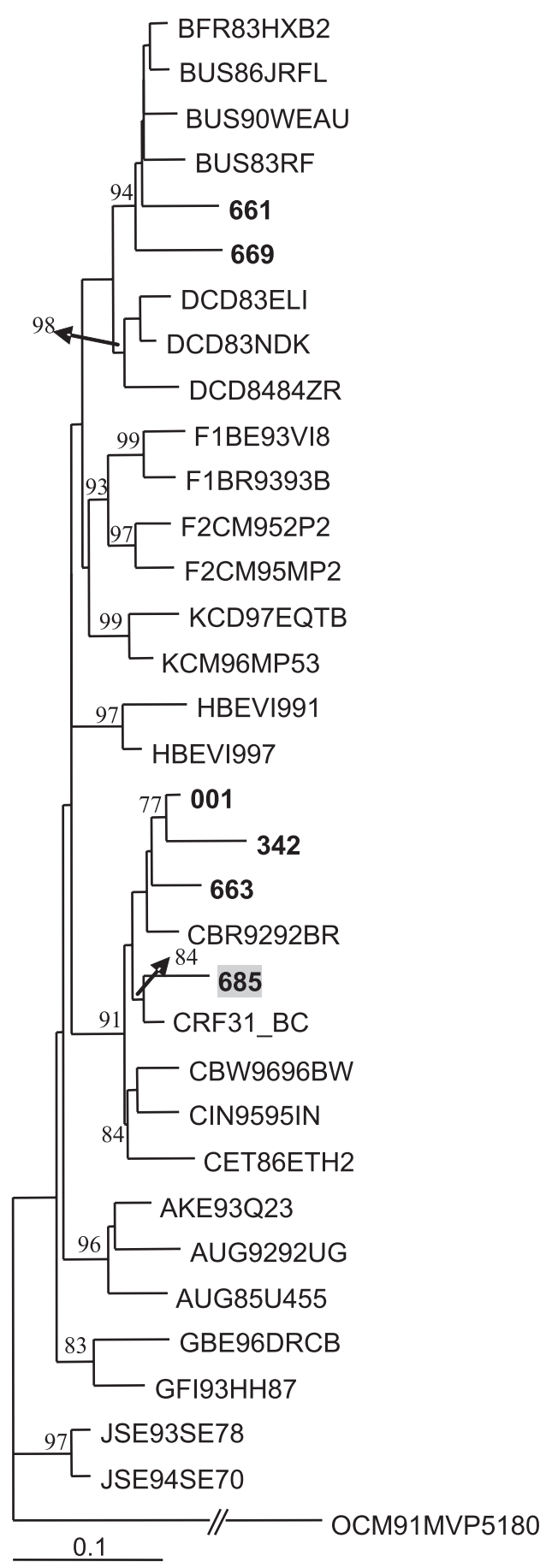

Figure 2. Schematic structure of the $\mathrm{BC}$ recombinant virus genome that we analyzed. Breakpoints were determined after re-analysis of the original sequences by the Simplot program.

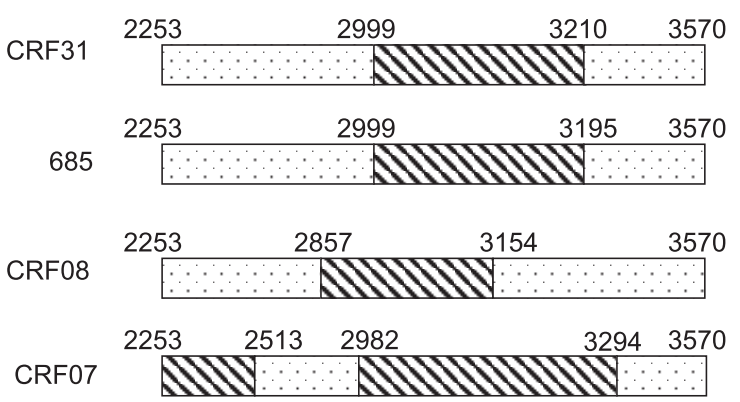

$\therefore$ HIV-1 C
HIV-1 B

the phylogenetic robustness of the clusters. The sequence OCM91MVP5180 (GenBank accession no. L20571) was used as an outgroup. The recombination pattern was determined by bootscanning analysis with SIMPLOT 2.5 software [17].

Subtype analyses screening of the index case showed a $\mathrm{BC}$ recombinant virus. In phylogenetic analysis, the sequence clustered with CRF31_BC on a neighbor joining tree, supported by a bootstrap value of $84 \%$, whereas the remaining clade $\mathrm{B}$ and $\mathrm{C}$ pol fragments grouped with the respective HIV1 subtype references (Figure 1). Bootscanning analysis showed that the sample shares the same breakpoints in pol region as CRF31_BC (Figures 2 and 3).

Phylogenetic analysis suggests that this child was infected by a BC recombinant virus. HIV-1 subtype $C$ circulates at a low rate in São Paulo and Rio de Janeiro, being found in approximately $1 \%$ of HIV-1 infected individuals [18,19]. We believe that this low frequency of HIV-1 C diminished the chance for recombinant events between HIV-1 subtype B and C.

In São Paulo state, BF mosaics are frequent $[6,20]$, but BC recombinants are very rare, especially in individuals infected more than 10 years ago, such as the child described here.

This fact may reflect that CRF31_BC is emerging in our state, but we can not be sure that the mother was indeed infected in São Paulo. However, as the mode of transmission is known, this case indicates that this $\mathrm{BC}$ recombinant has been circulating in São Paulo since 10 years ago, much longer than previously thought.

Understanding the causes of the successful spread of CRF31_BC in the southern part of the country, as well as monitoring the entry of this CRF into other regions of the country is important for public health strategies in Brazil, shedding light on aspects of virus fitness and niche competition between different HIV strains. 
Figure 3. Bootscanning analysis of the partial pol region of the $\mathrm{BC}$ recombinant virus genome that we analyzed. Horizontal axes represent nucleotide position in this region, whereas vertical axes depict bootscanning values (\%) that support the grouping of the isolate with each HIV-1 subtype.
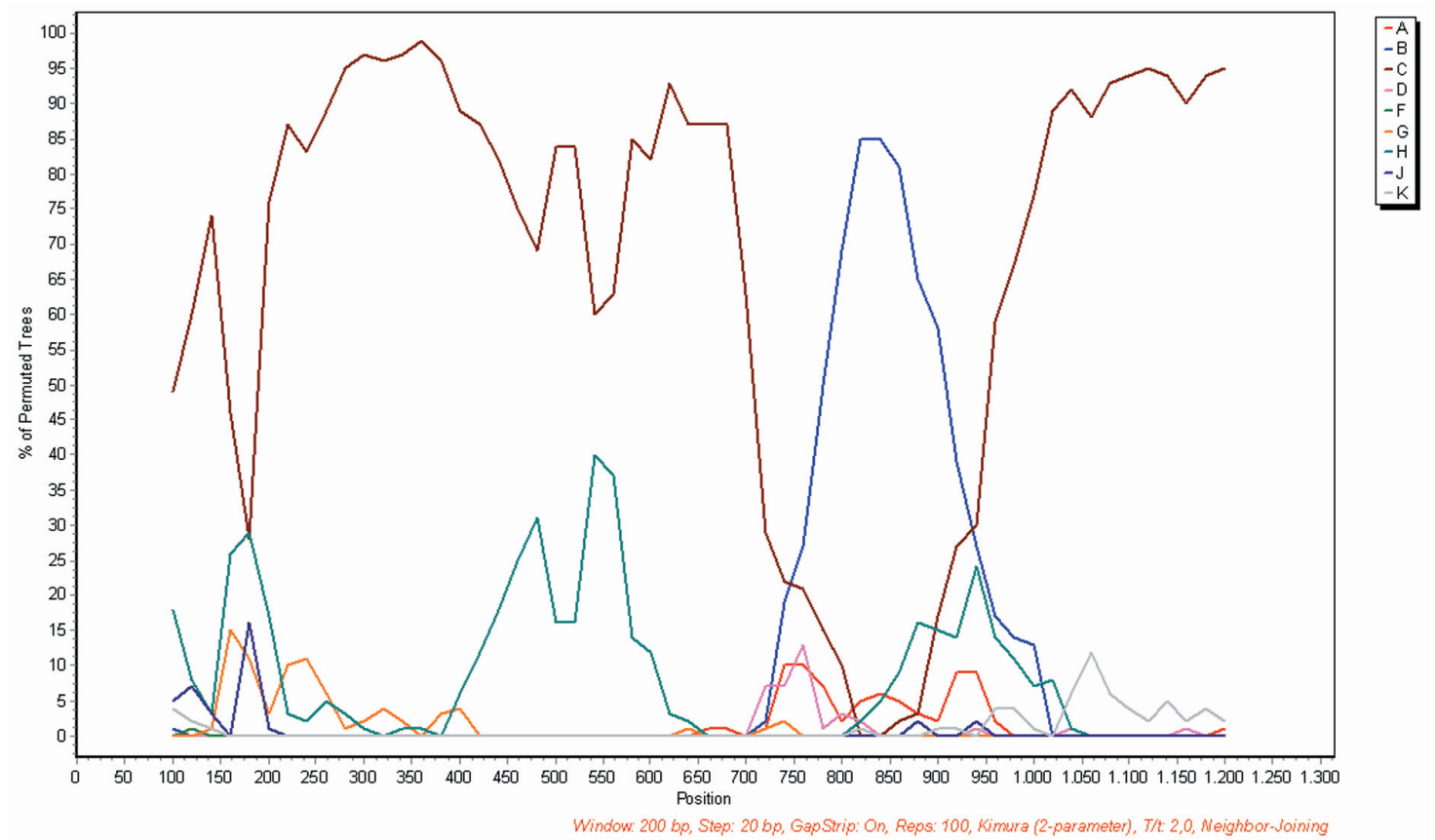

\section{References}

1. UNAIDS/WHO. Joint United Nations Programme on HIV/AIDS (UNAIDS)/World Health Organization (WHO). 2007 AIDS Epidemic Update.

2. Brasil. Ministério da Saúde. Secretaria de Vigilância em Saúde. Programa Nacional de DST e AIDS. Boletim Epidemiológico DST/AIDS. Ano III, $n^{\circ} 01-01^{\mathrm{a}}$. à 26 $6^{\mathrm{a}}$. Semanas Epidemiológicas-janeiro a junho de 2007. Brasília (DF); 2007.

3. Robertson DL, Anderson JP, Bradac JA et al. HIV-1 Nomenclature Proposal. Science 2000;288:55-6.

4. Los Alamos National Laboratory. HIV Sequence database. [http:/ /www.hiv.lanl.gov/content/sequence/HIV/CRFs/CRFs.html]2007.

5. Morgado M.G., Sabino E.C., Shpaer E.G., et al. V3 region polymorphisms in HIV-1 from Brazil: Prevalence of subtype B strains divergent from North American/European prototype and detection of subtype F. AIDS Research and Human Retroviruses 1994;10:569-76.

6. Sabino E.C., Shaper E.G., Morgado M.G., et al. Identification of human immunodeficiency virus type 1 envelope genes recombinant between subtypes $\mathrm{B}$ and $\mathrm{F}$ in two epidemiologically linked individuals from Brazil. J Virol 1994;68:6340-6.

7. Bongertz V., Bou-Habib D.C., Brígido L.F., et al. HIV-1 Diversity in Brazil: Genetic, Biologic, and Immunologic Characterization of HIV-1 Strains in Three Potential HIV Vaccine Evaluation Sites. J Acquir Immune Defic Syndr 2000;23:184-93.

8. Brindeiro R.M., Diaz R.S., Sabino E.C., et al. Brazilian Network for HIV Drug Resistance Surveillance (HIV-BresNet): a survey of chronically infected individuals. AIDS 2003;17:1063-9.

9. Csillag C. HIV-1 subtype C in Brazil. Lancet 1994;344:1354.

10. Brígido L.F., Nunes C., Oliveira C.M., et al. HIV-1 subtype C and $\mathrm{CB}$ pol recombinants prevail at the cities with the highest AIDS prevalence rate of Brazil. AIDS Res Hum Retroviruses 2007;23:1579-85.

11. Bello $\mathrm{G}$, Passaesa $\mathrm{CPB}$, Guimarães $\mathrm{ML}$ et al. Origin and evolutionary history of HIV-1 subtype C in Brazil. AIDS 2008;22:1993-2000.
12. Soares M.A., Oliveira T., Brindeiro R.M., et al. S specific subtype $\mathrm{C}$ of human immunodeficiency virus type 1 circulates in Brazil. AIDS 2003; $17: 11-21$.

13. Santos A.F., Sousa T.M., Soares E.A., et al. Characterization of a new circulating recombinant form comprising HIV1 subtype $\mathrm{C}$ and $\mathrm{B}$ in southern Brazil. AIDS $2006 ; 20: 2011-9$

14. Oliveira T., Deforche K., Cassol S., et al. An Automated Genotyping System for Analysis of HIV-1 and other Microbial Sequences. Bioinformatics 2005;21:3797-800.

15. Swofford D. Phylogenetic Analysis with Parsimony (and other Methods), version 4.0b2a. Sinauer Associates Inc., Sunderland, MA. 1999.

16. Posada D., Crandall K.A., MODELTEST: testing the model of DNA substitution. Bioinformatics 1998; 14:817-8.

17. Salminen M.O., Carr J.K., Burke D.S., McCutchan F.E. Identification of breakpoints in intergenotypic recombinants of HIV type 1 by bootscanning. AIDS Res Hum Retroviruses 1995; $11: 1423-5$.

18. Machado E.S., Lambert J.S., Watson D.C., et al. Genotypic resistance and HIV-1 subtype in Brazilian children on dual and triple combination therapy. J Clin Virol 2004;30:24-31.

19. Brindeiro P.A., Brindeiro R.M., Mortensen C., et al. Testing Genotypic and Fenotypic Resistance in Human Immunodeficiency Virus Type 1 Isolates of Clade B and Other Clades from Children Failing Antiretroviral Therapy. J Clin Microbiol 2002;40:4512-9.

20. Brígido L.F., Franco H.M., Custódio R.M., et al. Molecular characteristics of HIV type 1 circulating in São Paulo, Brazil. AIDS Res Hum Retroviruses 2005;21:673-82.

21. Rhodes T., Wargo H., Hu W.S. High rates of human immunodeficiency virus type 1 recombination: nearrandom segregation of markers one kilobase apart in one round of viral replication.J Virol 2003;77(20):11193-200. 